\title{
gु \\ Transition from hydrodynamic to viscoelastic propagation of sound in molten $\mathrm{RbBr}$
}

\author{
F. Demmel, ${ }^{1,}{ }^{*}$ D. Szubrin, ${ }^{2}$ W. C. Pilgrim, ${ }^{2}$ A. De Francesco,${ }^{3}$ and F. Formisano ${ }^{3}$ \\ ${ }^{1}$ ISIS Facility, Rutherford Appleton Laboratory, Didcot OX11 0QX, United Kingdom \\ ${ }^{2}$ Fachbereich Chemie, Philipps-Universität Marburg, 35032 Marburg, Germany \\ ${ }^{3}$ CNR-IOM clo OGG Institut Laue-Langevin, 38042 Grenoble, France \\ (Received 10 April 2015; published 9 July 2015)
}

\begin{abstract}
Inelastic neutron scattering was applied to measure the acoustic-type excitations in the molten alkali halide rubidium bromide. For molten $\mathrm{RbBr}$ neutron scattering is mainly sensitive to the number density fluctuation spectrum and is not influenced by charge fluctuations. Utilizing a dedicated Brillouin scattering spectrometer, we focused on the small-wave-vector range. From inelastic excitations in the spectra a dispersion relation was obtained, which shows a large positive dispersion effect. This frequency enhancement is related to a viscoelastic response of the liquid at high frequencies. Towards small wave vectors we identify the transition to hydrodynamic behavior. This observation is supported by a transition of the sound velocity from a viscoelastic enhanced value to the adiabatic speed of sound for the acoustic-type excitations. Furthermore, the spectrum transforms into a line shape compatible with a prediction from hydrodynamics.
\end{abstract}

DOI: 10.1103/PhysRevE.92.012307

PACS number(s): 05.20.Jj, 61.05.F-

\section{INTRODUCTION}

Liquids are characterized by disorder and strong correlations between the particles, which separates a liquid from the crystal and the gas. These strong correlations combined with disorder hamper the progress for a comprehensive theoretical description. In particular, a precise understanding of the singleparticle and collective dynamics in such strongly correlated systems is a challenge for statistical mechanics. To guide and support this endeavor input from experiment is mandatory. The fruitful exchange during the past decades provided good progress in the understanding of liquid dynamics in some fields. In particular, the liquid dynamics of monatomic systems benefited with some remarkable successes, like the experimental verification of the long time-tail in the decay of correlation functions [1] or the enhancement of the sound velocity of acoustic-type modes above the adiabatic sound velocity, the so-called positive dispersion [2]. The detected excitations can be understood as high-frequency propagating sound modes, due to their linear dispersion at small values of the exchanged momentum $Q$. The advent of the inelastic x-ray scattering technique provided new insight into the relaxation dynamics of density fluctuations and gave evidence for a further decay process of the acoustic-type excitations (for a recent review, see Ref. [3]).

For binary liquids the most remarkable predictions have been the existence of optic-type modes and the prediction of fast sound. In both cases there is a scarcity of experimental work to support these predictions from molecular dynamics (MD) simulations. For example, evidence for optic-type modes has been reported for liquid deuterium fluoride [4] and molten $\mathrm{NaI}$ [5]. More recently, the coexistence of a low-energy

\footnotetext{
*franz.demmel@stfc.ac.uk
}

Published by the American Physical Society under the terms of the Creative Commons Attribution 3.0 License. Further distribution of this work must maintain attribution to the author(s) and the published article's title, journal citation, and DOI. acoustic mode and a high-energy opticlike excitation has been reported by investigating a liquid alloy $\left(\mathrm{Li}_{30} \mathrm{Bi}_{70}\right)$ [6] and evidence for two collective modes in binary alloys with competing interactions has been reported [7]. The fast sound prediction [8], an excitation of the light element on the background of the heavy component in a disparate mass fluid, ignited a frantic search in particular in water dynamics [9-11], despite the fact that early on the sound velocity enhancement has been explained by a viscoelastic reaction of the liquid [12]. For rare-gas mixtures evidence exists for fast sound from experiment [13]. However, even the less-spectacular acoustictype excitations have not been studied experimentally in such detail in binary systems as is the case in monatomic liquids. One reason for this might be the wealth of information one gets in a single scattering experiment, which is often difficult or even impossible to separate into the individual components of interest. One of the model binary systems are molten alkali halides due to their simple Coulomb interaction, which renders molten salts one of the most simulated binary liquids. The study of binary ionic liquids and, as their prototypes, molten alkali halides, has a long tradition in theory, experiment, as well as in simulation [14-16].

Besides the interest in understanding the fundamental principles in the dynamics of a binary Coulomb liquid, molten salts have gained attractiveness as useful materials, for example, in energy research because of their potential use as heat storage in modern solar power plants or as coolant in next-generation nuclear reactors. One of the questions that link application-oriented research with fundamental physics is how the bulk properties of these systems are related to the particle behavior on microscopic time and length scales. For this, however, detailed knowledge of the microscopic structural and dynamic properties is indispensable.

From a structural point of view, Coulomb liquids exhibit short-range order due to the electrical charge, which shows an alternation in charge of the successive coordination shells around a central ion. Neutron-scattering experiments, applying isotopic substitution, measured the partial structure factors [17] and the derived pair distribution functions show maxima at alternating distances up to a radius of about $12 \AA$ [18]. 
This solidlike structural arrangement is probably responsible for the peculiar dynamic response, which resembles the solid state, too.

One prominent feature in the dynamics is the prediction of optic-type modes as was shown in the pioneering computer simulation of a simple molten salt by Hansen and McDonald about four decades ago [19]. On the other hand, their data gave no hints for propagating acoustic modes in the spectra, a result which was attained later several times in MD simulations [20,21]. With inclusion of ion polarization, as was shown for $\mathrm{NaI}$, indications for propagating density fluctuations were found as shoulders at the smallest $Q$ vectors [22]. The fact that polarization might play a fundamental role in alkali halide dynamics was already recognized after the first phonon measurements with neutrons on alkali halide crystals [23] Later MD simulations with modified rigid ion potentials identified acoustic-type excitation shoulders in the mass density correlation function spectra, modeling molten NaI [24]. At the smallest simulated wave vector $Q=0.175 \AA^{-1}$ a sound velocity similar to the adiabatic sound velocity was reported.

Neutron-scattering groups have intensively investigated the ion dynamics in molten salts and searched for the expected optic modes [25]. However, inelastic neutron-scattering experiments are always restricted in the kinematic accessible range. Therefore most of the available experimental data on dispersing excitations were obtained at momentum transfers beyond $1 \AA^{-1}[18,25,26]$. In addition, no evidence of propagating acoustic-like modes could be observed in the spectra directly, because at these relatively large $Q$ values the lifetime of an acoustic mode might be already too short. However, Brillouin light scattering demonstrated propagating acoustic modes in the $\mathrm{GHz}$ regime in molten $\mathrm{KCl}$ [27]. The measured sound velocity is nearly unchanged compared to the results of ultrasound methods [28].

Finally, the new technique of high-resolution inelastic $\mathrm{X}$-ray scattering demonstrated acoustic-type modes in molten alkali halides beyond the hydrodynamic regime [29-32]. Surprisingly, the acoustic-type dispersion in molten $\mathrm{NaCl}$ and $\mathrm{KCl}$ resembled very well the dispersions of the respective metal cations. It was suggested that at high frequencies a decoupling of the cation from the anion subsystem may occur. At large $Q$ vectors, when short-distance correlations are investigated, the dynamics of the different modes mirrors the partial dynamics of the individual elements. However, how the collective dynamics evolves in the intermediate wave-vector range is still under scrutiny, in particular for ionic liquids, where, in addition, optic-type excitations emerge.

Here we present an investigation on the acoustic modes of molten rubidium bromide with an emphasis on the smallwave-vector range studied by inelastic neutron scattering. Four decades ago molten $\mathrm{RbBr}$ was measured by use of inelastic neutron scattering [26]. At that time no data were reported for wave vectors $Q<1 \AA^{-1}$. A similar wave-vector range was explored on a $\mathrm{Rb}-\mathrm{RbBr}$ mixture demonstrating dispersion relations from current correlation functions [33]. A classical MD simulation on a rigid ion model of molten $\mathrm{RbBr}$ gave evidence for an enhanced sound velocity and hence for a departure from hydrodynamics [34]. The masses of both ions are very similar and therefore no potential transition to a fast-sound-type excitation will occur in this system. In
TABLE I. Neutron coherent scattering lengths, cross sections, and absorption cross sections of $\mathrm{Rb}$ and $\mathrm{Br}$ are presented.

\begin{tabular}{llccc}
\hline \hline Ion & $\mathrm{b}(\mathrm{fm})$ & $\sigma_{\text {coh }}($ barn $)$ & $\sigma_{\text {inc }}($ barn $)$ & $\sigma_{\text {abs }}($ barn $)$ \\
\hline $\mathrm{Rb}$ & 7.09 & 6.3 & 0.04 & 0.38 \\
$\mathrm{Br}$ & 6.795 & 5.8 & 0.1 & 6.9 \\
\hline \hline
\end{tabular}

fact, the transition from the viscoelastic microscopic response to the hydrodynamic regime in a molten salt is the focus of this investigation. In noble gases the departure from the hydrodynamic behavior has been examined experimentally $[35,36]$ and numerically [37].

\section{FORMAL FRAMEWORK}

The neutron interaction with nuclei in nonmagnetic materials can be decomposed in the sum of a coherent and an incoherent contribution. The measured total double differential cross section can therefore be written as:

$$
\frac{d^{2} \sigma}{d \Omega d \omega}=\frac{d^{2} \sigma^{\mathrm{coh}}}{d \Omega d \omega}+\frac{d^{2} \sigma^{\mathrm{inc}}}{d \Omega d \omega} .
$$

The incoherent cross section connects with the selfcorrelations of a single particle and the coherent cross section provides information on correlations between the particles. In a multicomponent system the double differential cross sections per atom can be written in the following form [26]:

$$
\frac{d^{2} \sigma^{\mathrm{coh}}}{d \Omega d \omega}=\frac{k_{f}}{k_{i}} \sum_{a} \sum_{b} b_{a} b_{b}\left(c_{a} c_{b}\right)^{1 / 2} S_{a b}(Q, \omega)
$$

and

$$
\frac{d^{2} \sigma^{\mathrm{inc}}}{d \Omega d \omega}=\frac{k_{f}}{k_{i}} \sum_{a} \frac{\sigma^{\mathrm{inc}}}{4 \pi} c_{a} S_{a}^{s}(Q, \omega),
$$

where $k_{f}$ and $k_{i}$ are the scattered and incident neutron wave vectors, $b_{a}$ denote the scattering lengths, and $c_{a}=N_{a} / N$ the respective atomic concentrations. The total differential cross section is then given by a cross-section-weighted sum of these two scattering functions $S_{a b}(Q, \omega)$ and $S_{a}^{S}(Q, \omega)$. This definition follows the Ashcroft-Langreth description of partial structure factors, which ensures that $a=b$ structure factors converge to 1 for large $Q$ vectors [38].

In a binary system the measured intensity will consist of five different contributions: two incoherent dynamic structure factors and three partial dynamic structure factors from the correlations of the particles. The incoherent cross sections of $\mathrm{Rb}$ and $\mathrm{Br}$ are practically negligible (see Table I) $[39,40]$. Hence we can write the double differential cross section for $\mathrm{RbBr}$ as a sum over the three partial dynamic scattering functions taking into account the concentrations $c_{a, b}=0.5$ :

$$
\begin{aligned}
\frac{d^{2} \sigma}{d \Omega d \omega}= & \frac{k_{f}}{k_{i}} \frac{1}{2}\left[b_{\mathrm{Rb}}^{2} S_{\mathrm{RbRb}}(Q, \omega)+2 b_{\mathrm{Rb}} b_{\mathrm{Br}} S_{\mathrm{RbBr}}(Q, \omega)\right. \\
& \left.+b_{\mathrm{Br}}^{2} S_{\mathrm{BrBr}}(Q, \omega)\right] .
\end{aligned}
$$

The dynamic structure factors are weighted by the scattering lengths $b$ (note the subscript $b$ is used to denote the scattering length and an atomic species). The partial dynamic structure 
factors $S_{a b}(\mathbf{Q}, \omega), a, b=\mathrm{Rb}, \mathrm{Br}$ are the spectral densities of the fluctuations of the microscopic particle densities $\rho_{a}(\mathbf{r}, t)=$ $\Sigma_{i a} \delta\left[\mathbf{r}-\mathbf{r}_{i a}(t)\right]$, whose components in the reciprocal space are

$$
\rho_{a}(\mathbf{Q}, t)=\sum_{i a=1}^{N_{a}} \exp \left[-i \mathbf{Q} \cdot \mathbf{r}_{i a}(t)\right],
$$

where $a$ can be either + or - and $\mathbf{r}_{i a}(t)$ is the position at time $t$ of the ion $i_{a}$ of species $a$. Their time correlations are the partial intermediate scattering functions

$$
F_{a b}(Q, t)=\frac{1}{N} \frac{\left\langle\rho_{a}(\mathbf{Q}, t) \rho_{b}(-\mathbf{Q}, 0)\right\rangle}{\sqrt{c_{a} c_{b}}},
$$

where $N=N_{+}+N_{-}, c_{a}=N_{a} / N$ and the brackets denote the ensemble average over equilibrium configurations.

The partial number density is not the only suitable variable to describe a dynamic system. Other dynamical variables like total number density $N(r, t)$, mass density $M(r, t)$, or charge density $Z(r, t)$ can be defined and provide a more direct link to physical quantities, like, e.g., acoustic and optic modes. For a two particle system with equal charges $\pm 1 e$ the microscopic charge density can be written as:

$$
Z(\mathbf{r}, \mathbf{t})=\frac{1}{e}\left[Z_{a} \rho_{a}(\mathbf{r}, t)+Z_{b} \rho_{b}(\mathbf{r}, t)\right]=\rho_{a}(\mathbf{r}, t)-\rho_{b}(\mathbf{r}, t)
$$

and $\rho_{N}(r, t)=\rho_{a}(\mathbf{r}, t)+\rho_{b}(\mathbf{r}, t)$. Then number density $S_{N N}(Q, \omega)$ and charge density dynamic structure factor $S_{Z Z}(Q, \omega)$ and their cross correlation $S_{N Z}(Q, \omega)$ can be obtained as linear combinations from the partial dynamic structure factors:

$$
\begin{aligned}
& \mathrm{S}_{N N}(Q, \omega)=\frac{1}{2}\left[S_{a a}(Q, \omega)+2 S_{a b}(Q, \omega)+S_{b b}(Q, \omega)\right], \\
& \mathrm{S}_{Z Z}(Q, \omega)=\frac{1}{2}\left[S_{a a}(Q, \omega)-2 S_{a b}(Q, \omega)+S_{b b}(Q, \omega)\right],
\end{aligned}
$$

and

$$
\mathrm{S}_{N Z}(Q, \omega)=\frac{1}{2}\left[S_{a a}(Q, \omega)-S_{b b}(Q, \omega)\right] .
$$

Mass correlation functions can be defined, too. However, with similar masses, as is the case for $\mathrm{Rb}$ and $\mathrm{Br}$, there is practically no difference to the number density dynamic structure factor. Finally, we obtain the measured double differential cross section Eq. (4) as a sum over number density, charge density, and their cross correlation functions, taking into account the scattering lengths:

$$
\begin{aligned}
\frac{d^{2} \sigma}{d \Omega d \omega}= & \frac{k_{f}}{k_{i}} \frac{1}{4}\left[\left(b_{\mathrm{Rb}}+b_{\mathrm{Br}}\right)^{2} S_{N N}(Q, \omega)+2\left(b_{\mathrm{Rb}}^{2}-b_{\mathrm{Br}}^{2}\right)\right. \\
& \left.\times S_{N Z}(Q, \omega)+\left(b_{\mathrm{Rb}}-b_{\mathrm{Br}}\right)^{2} S_{Z Z}(Q, \omega)\right] .
\end{aligned}
$$

The number density fluctuations are related to acoustic-type excitations and charge fluctuations to optic-type modes. This choice of dynamic variables thus allows us to make direct contact with acoustic and optic modes in the molten salt. The scattering lengths of rubidium and bromine are nearly equal (see Table I), which leaves us only with one term, the number density dynamic scattering function $S_{N N}(Q, \omega)$. Hence a neutron-scattering experiment on molten $\mathrm{RbBr}$ will mainly be sensitive to the acoustic-type excitations. At large momentum transfer the dynamics of the individual ions will dominate the signal and a suitable characterization might be given by Eq. (4). With similar masses of the ions the two predicted modes should coincide for $\mathrm{RbBr}$, hence we expect to observe one acoustic-type mode with a transition from hydrodynamic to microscopic propagation of sound. In the scattering experiment on $\mathrm{RbBr}$ the neutrons are uniquely sensitive to one dynamical quantity, namely the number density fluctuations. As a consequence, the data modeling can be based on functional forms as in the monatomic case.

Another important dynamic quantity is the current. Similarly to the partial local number densities the partial local velocities are defined as

$$
\mathbf{v}_{a}(\mathbf{r}, t)=\sum_{i=1}^{N_{a}} \mathbf{v}_{a i}(t) \delta\left[\mathbf{r}-\mathbf{r}_{a i}(t)\right] .
$$

The product with the corresponding partial local number density yields the partial local current $\mathbf{j}_{a}(\mathbf{r}, t)=\mathbf{v}_{a}(\mathbf{r}, t) \rho(\mathbf{r}, t)$, whose space Fourier transform is

$$
\mathbf{j}_{a}(\mathbf{Q}, t)=\sum_{i=1}^{N_{a}} \mathbf{v}_{a i}(t) e^{-i \mathbf{Q} \cdot \mathbf{r}_{a i}(t)} .
$$

The partial local current is related to the partial local number density by the continuity equation. Hence, the partial longitudinal current correlation function is directly related to $S(\mathbf{Q}, \omega)$,

$$
J(\mathbf{Q}, \omega)=\frac{\omega^{2}}{Q^{2}} S(\mathbf{Q}, \omega) .
$$

This direct relation implies that both the dynamic structure factor and the current spectra contain the same amount of information on the inelastic dynamics. However, the $\omega^{2}$ behavior imposes the presence of a peak in the spectra of the current correlation function and inelastic peaks which are well visible for any wave vector even appear when no excitation is obvious in the $S(Q, \omega)$ spectra. For well-defined inelastic excitations both descriptions deliver the same dispersion. We have applied both approaches to investigate the dynamic behavior of molten $\mathrm{RbBr}$.

At times longer than typical atomic collision times and wavelengths much longer than typical interparticle distances, hydrodynamics predicts a triplet structure for the dynamic structure factor of a monatomic liquid [41]. The solution of the linearized Navier-Stokes equations yields two Brillouin lines dispersing with the sound velocity and one quasielastic Rayleigh line. The solution for a binary liquid is more complex, in particular for the Rayleigh line. If thermal relaxation and interdiffusion are independent, the spectrum of a binary liquid might be written as a sum over four Lorentzian lines [41]. In diluted systems such an approach is justified, but it is not known whether in a dense binary liquid with equal concentrations of the two types of particles this ansatz is still useful. Furthermore, a fit of a single Rayleigh line with two Lorentzians of similar width tends to be ambiguous.

The Mori-Zwanzig formalism provides a powerful framework to define the dynamics of dense liquids [42]. Herein the dynamics is defined within a generalized Langevin equation for the time-dependent correlation function which, Laplace transformed, can be presented in a continued fraction 
representation:

$$
F(Q, z)=\frac{S(Q)}{z+\frac{\omega_{0}^{2}}{z+K(Q, z)}},
$$

where $\omega_{0}^{2}$ is the normalized second frequency moment of $S(Q, \omega)$ and $K(Q, z)$ is the Laplace transform of the memory function $K(Q, t)$. Theoretically the normalized second frequency moment is given by the expression $\omega_{0}^{2}=\frac{k_{B} T}{m S(Q)} Q^{2}[42]$. The dynamic structure factor is then related to $F(Q, z=i \omega)$ through:

$$
\frac{S(Q, \omega)}{S(Q)}=\frac{1}{\pi} \operatorname{Re}\left[\frac{F(Q, z)}{S(Q)}\right] .
$$

The generalized Langevin equation with memory is an exact equation, which can be derived within the projection operator formalism from the Liouville equation. All the unknowns in the dynamics is packed into the memory function. Now we closely follow the representation by Balucani and Zoppi [42] to present, at the end, the final functional fit forms for the viscoelastic and the hydrodynamic cases derived from the memory function approach, which are rarely documented.

A simple approach uses an exponential decaying memory function $K(Q, t)=K(Q, 0) \exp [-t / \tau(Q)]=\left(\omega_{l}^{2}-\right.$ $\left.\omega_{0}^{2}\right) \exp [-t / \tau(Q)]$, where the relaxation time $\tau$ is known as the Maxwell relaxation time. $\omega_{l}^{2}(Q)$ is the ratio of the fourth to the second frequency moment of the dynamic structure factor. This single exponential decay in the memory function is the so-called viscoelastic model. That ansatz can be solved and one obtains eventually:

$$
S(Q, \omega)=\frac{S(Q)}{\pi} \frac{\left(\omega_{l}^{2}-\omega_{0}^{2}\right) \tau \omega_{0}^{2}}{\omega^{2} \tau^{2}\left(\omega^{2}-\omega_{l}^{2}\right)^{2}+\left(\omega^{2}-\omega_{0}^{2}\right)^{2}} .
$$

This result was already obtained by Lovesey some time ago [43]. Besides the structure factor $S(Q)$ and the relaxation time $\tau(Q)$ there appear two further parameters: $\omega_{0}^{2}(Q)$ and $\omega_{l}^{2}(Q)$. A helpful approximation for $\omega_{l}^{2}(Q)$, which we will apply, was provided by Hubbard and Beeby [44]:

$$
\omega_{l}^{2}=\omega_{E}^{2}\left[1-3 \frac{\sin (Q \sigma)}{Q \sigma}-6 \frac{\cos (Q \sigma)}{(Q \sigma)^{2}}+6 \frac{\sin (Q \sigma)}{(Q \sigma)^{3}}\right]
$$

with $\omega_{E}$ as the Einstein frequency and $\sigma$ a hard sphere parameter of the particles. From Eq. (17) one can consider two limiting cases: In the high-frequency case $\omega \tau>>1$ the poles of Eq. (17) are determined by the $\omega_{l}$ values. At high frequencies the instantaneous response of the system is probed and a high-frequency phase velocity can be defined: $c_{\infty}(Q)=\frac{\omega_{l}}{Q}$. The liquid responds as it is a frozen solidlike system with an enhanced sound velocity for the acoustic-type excitations. For small frequencies $\omega \tau<<1$ the sound propagation is defined by the isothermal sound velocity $c(Q)=\frac{\omega_{0}}{Q}$. This transition in the dynamics from viscous to elastic behavior lies at the heart of this viscoelastic model.

This single-exponential ansatz was successfully applied to describe the collective dynamics of liquid alkali metals, for example, liquid cesium and rubidium $[2,45,46]$. For alkali metals the contribution of thermal relaxations, related to the ratio of the specific heats $c_{p} / c_{v}=1.1$, is quite small and therefore can be neglected. However, even for the monatomic case it was argued that a two-exponential relaxation process in the second-order memory function $K(Q, t)$ improves the data fit [3]. In MD simulations on a Lennard-Jones fluid, Levesque et $\mathrm{al}$. reported the need for a second relaxation time in the memory function to describe the spectra of transverse excitations [47]. In a simulation on a molten salt, mimicking $\mathrm{NaCl}$, it was noted that the modeling of some dynamical quantities needed a two-times model within the memory function [20].

For binary salts the ratio of specific heats $\gamma=c_{p} / c_{v}$ is no longer negligible. For molten $\mathrm{KBr} \gamma=1.42$ and for $\operatorname{CsBr} \gamma=$ 1.63 were reported [48], so we estimate that $\gamma=c_{p} / c_{v} \approx 1.5$ for the intermediate case of molten $\mathrm{RbBr}$. Hence we add a second relaxation channel to the memory function:

$$
\begin{aligned}
K(Q, t)= & \left(\omega_{l}^{2}-\gamma \omega_{0}^{2}\right) \exp [-t / \tau(Q)] \\
& +(\gamma-1) \omega_{0}^{2} \exp \left(-D_{T} Q^{2} t\right) .
\end{aligned}
$$

$D_{T}$ is the thermal diffusion coefficient which is related to the thermal conductivity $\lambda: D_{T}=\lambda / n c_{p}$ and $n$ is the particle density. After a Laplace transform we obtain:

$$
\frac{F(Q, z)}{S(Q)}=\frac{1}{z+\frac{\omega_{0}^{2}}{z+\frac{\Delta_{1}}{z+\Gamma_{1}}+\frac{\Delta_{2}}{z+\Gamma_{2}}}}
$$

with $\Delta_{1}=\omega_{l}^{2}-\gamma \omega_{0}^{2}, \Delta_{2}=(\gamma-1) \omega_{0}^{2}, \Gamma_{1}=1 / \tau(Q)$, and $\Gamma_{2}=D_{T} Q^{2}$. Here $\tau(Q)$ is the structural, viscoelastic relaxation time and $\tau_{T}=1 / \Gamma_{2}$ is the thermal relaxation time.

After some simplifying we arrive at the following expression for the two-time viscoelastic model:

$$
\frac{F(Q, z)}{S(Q)}=\frac{z^{3}+z^{2} f_{1}+z f_{2}+f_{3}}{z^{4}+z^{3} f_{1}+z^{2} g_{2}+z g_{3}+g_{4}},
$$

with $f_{1}=\Gamma_{1}+\Gamma_{2}, \quad f_{2}=\Gamma_{1} \Gamma_{2}+\Delta_{1}+\Delta_{2}, \quad f_{3}=\Delta_{1} \Gamma_{2}+$ $\Delta_{2} \Gamma_{1}, \quad g_{2}=\Gamma_{1} \Gamma_{2}+\Delta_{1}+\Delta_{2}+\omega_{0}^{2}, \quad g_{3}=\Delta_{1} \Gamma_{2}+\Delta_{2} \Gamma_{1}+$ $\omega_{0}^{2}\left(\Gamma_{1}+\Gamma_{2}\right)$, and $g_{4}=\omega_{0}^{2} \Gamma_{1} \Gamma_{2}$.

A similar derivation can be found in Ref. [49] with the difference that these authors then write the fraction of two polynomials as a sum of partial fractions of real and complex roots, which represent exponentially decaying intermediate scattering functions in the time domain. That approach, resulting in a sum of Lorentzians in the frequency space, leads then to the so-called generalized hydrodynamics representation. After more algebra we arrive at (more details are presented in the Appendix):

$$
\frac{S(Q, \omega)}{S(Q)}=\frac{1}{\pi} \frac{\omega^{4}\left(f_{1} g_{2}+f_{3}-g_{3}-f_{1} f_{2}\right)+\omega^{2}\left(f_{2} g_{3}-f_{1} g_{4}-f_{3} g_{2}\right)+f_{3} g_{4}}{\omega^{8}+\omega^{6}\left(f_{1}^{2}-2 g_{2}\right)+\omega^{4}\left(g_{2}^{2}+2 g_{4}-2 f_{1} g_{3}\right)+\omega^{2}\left(g_{3}^{2}-2 g_{2} g_{4}\right)+g_{4}^{2}} .
$$

This fit model has five parameters: $S(Q), \omega_{0}^{2}, \omega_{l}^{2}, \Gamma_{1}$, and $\Gamma_{2}$, which are all $Q$ dependent. 
Towards long wavelengths the relaxation time for the density fluctuations becomes much larger than the microscopic relaxation time of the memory function. Consequently, that relaxation time can be replaced by a $\delta$ function and the memory function becomes $K(Q, t)=2 \eta_{L} /(n m) Q^{2} \delta(t)+$ $(\gamma-1) \omega_{0}^{2} \exp \left(-D_{T} Q^{2} t\right)$ with the longitudinal viscosity $\eta_{L}=$ $\frac{4}{3} \eta_{S}+\eta_{B}$ and $m$ the mass of the particle. $\eta_{S}$ is the shear viscosity and $\eta_{B}$ is the bulk viscosity. A similar algebraic derivation delivers eventually:

$$
\frac{S(Q, \omega)}{S(Q)}=\frac{1}{\pi} \frac{\omega^{2}\left(f_{1} g_{2}-f_{2} g_{1}-g_{3}\right)+f_{2} g_{3}}{\omega^{6}+\omega^{4}\left(g_{1}^{2}-2 g_{2}\right)+\omega^{2}\left(g_{2}^{2}-2 g_{1} g_{3}\right)+g_{3}^{2}},
$$

with $\quad \Gamma_{L}=\eta_{L} / n m, \quad f_{1}=2 \Gamma_{L} Q^{2}+D_{T} Q^{2}, \quad f_{2}=$ $2 \Gamma_{L} D_{T} Q^{4}+(\gamma-1) \omega_{0}^{2}, \quad g_{2}=2 \Gamma_{L} D_{T} Q^{4}+\gamma \omega_{0}^{2}, \quad$ and $g_{3}=\omega_{0}^{2} D_{T} Q^{2}$. It has been shown [49] that this spectrum consists of a central Rayleigh line and two Brillouin lines which disperse with the adiabatic velocity of sound. In hydrodynamics density fluctuations are decaying macroscopically slow so they can be described using linearized Navier-Stokes equations from hydrodynamics, which was the first method to derive the time correlation function [50]. That model has four fit parameters: $S(Q), \omega_{0}^{2}$, $D_{T}$, and $\Gamma_{L}$. Here $c_{\text {ad }}=\gamma \frac{\omega_{0}}{Q}$ defines the propagation velocity of the acoustic modes and $\Gamma_{L}$ characterizes their damping. Note that fits with Eq. (23) have been performed but not with a three-Lorentzian model. We did not use the specific-heat ratio as an additional fit parameter. A constant $\gamma=1.5$ was used throughout the data analysis.

\section{EXPERIMENTAL DETAILS}

An inelastic neutron-scattering experiment on molten $\mathrm{RbBr}$ was performed at the BRISP spectrometer of the Institut Laue-Langevin (Grenoble, France). That spectrometer was specifically designed for small-angle spectroscopy with thermal neutrons [51] and its performance in this field recently has been demonstrated [52]. An incoming energy of $E_{i}=$ $51.9 \mathrm{meV}$ was used by exploiting the copper (111) reflection of the crystal monochromator. The sample-detector distance was $4 \mathrm{~m}$ with a maximum $2 \theta$ scattering angle of $14^{\circ}$. With an efficient collimation in place (honeycomb collimator [53]) spectra down to a scattering angle of $1^{\circ}$ can be measured. The sample was contained in a niobium cylindrical can and enclosed by electron beam welding. The can has a diameter of $28 \mathrm{~mm}$ and a height of $10 \mathrm{~mm}$ and was placed with its axis parallel to the neutron beam. The niobium windows of the can in-beam direction are only $200 \mu \mathrm{m}$ thick. A boron nitride mask shielded the outer parts of the cell from scattering. The can was installed in a standard furnace with niobium shields. Several scans on molten $\mathrm{RbBr}$, empty cell at high temperature, and a vanadium plate were performed. The total measurement time was about $50 \mathrm{~h}$ for the sample and the same amount was used for the empty can. The measurement temperature was $993 \mathrm{~K}$. The melting temperature of $\mathrm{RbBr}$ is $955 \mathrm{~K}$. The first peak of the partial structure factor is at about $Q=1.5 \AA^{-1}$ [54] and hence outside the accessible wave-vector range, which was limited to $0.2 \AA^{-1}<Q<1.1 \AA^{-1}$.
The data reduction included monitor normalization, subtraction of a time-of-flight background, angular integration and averaging, background subtraction, and conversion to energy space. A conversion to constant $Q$ spectra was then carried out. All of these analysis steps were performed using the lamp data reduction framework [55]. The vanadium scan delivered a Gaussian line shape with a full width at half maximum (FWHM) of $1.6 \mathrm{meV}$. In addition, the vanadium run was utilized to achieve a normalization in absolute units.

In scattering experiments higher-order scattering processes can spoil the measured signal. This is particularly important at small momentum vectors. Multiple scattering has been treated with a calculation of the twice-scattered neutrons in an efficient way for a prescribed single scattering law. This is performed by numerical evaluation of the analytically exact secondary scattering integral, taking into regard the proper kinematic restrictions. The same approach was used successfully for liquid rubidium [45]. The most important step in multiple scattering evaluation is to define the scattering law over the whole allowed kinematic range of higher-order scattering events, which prohibits the use of the parametrized measured signal. As input for the scattering function we use the coherent Lovesey model according to Eq. (17). The structure factor and the fourth frequency moment are necessary as input parameters. The structure factor is calculated using the hard-sphere Percus-Yevick approximation. For the hardsphere parameter $\sigma$ we use the arithmetic average of the ion diameters $\sigma=3.4 \AA$. For the normalized fourth moment $\omega_{l}^{2}$ the approximation from Hubbard and Beeby is applied according to Eq. (18). In order to determine the relaxation time $\tau$ we apply the recipe from Lovesey [43]:

$$
\frac{1}{\tau}=\frac{2}{\sqrt{\pi}} \sqrt{\omega_{l}^{2}-\omega_{0}^{2}}
$$

with the normalized second frequency moment $\omega_{0}^{2}$. We fitted the nonmultiple scattering corrected spectra with the Lovesey model according to Eq. (17). The resulting dispersion was used as input to fit the dispersion relation according to the approximation by Hubbard and Beeby and hence to derive the Einstein frequency. The Einstein frequency we obtained is $\omega_{E}=12 \times 10^{12} \mathrm{ps}^{-1}$. Then an input scattering function could be calculated over the whole accessible kinematic space in $Q$ and $\omega$. Only with a definition of the input $S(Q, \omega)$ over energies of twice the measured energy transfer and twice the maximum wave vector a reasonable description of two scattering events can be expected. Finally, the absolute value for the two-times scattering neutron signal is calibrated through a comparison between the single scattered calculated signal with the measured intensities (Fig. 1).

After subtracting the multiple scattering contribution we obtain fully corrected $S(Q, \omega)$ spectra. The spectra have been corrected for detailed balance by multiplication with $\exp \left(-\hbar \omega / 2 k_{B} T\right)$. At that high temperature $(T=993 \mathrm{~K})$ the correction is only a small effect and did not deliver symmetrical spectra. Therefore a sloping background was taken into account by adding a line to the fit model. This residual background probably stems from a not fully subtracted underlying background contribution in the time-of-flight signal. The sloping background increases towards smaller wave 


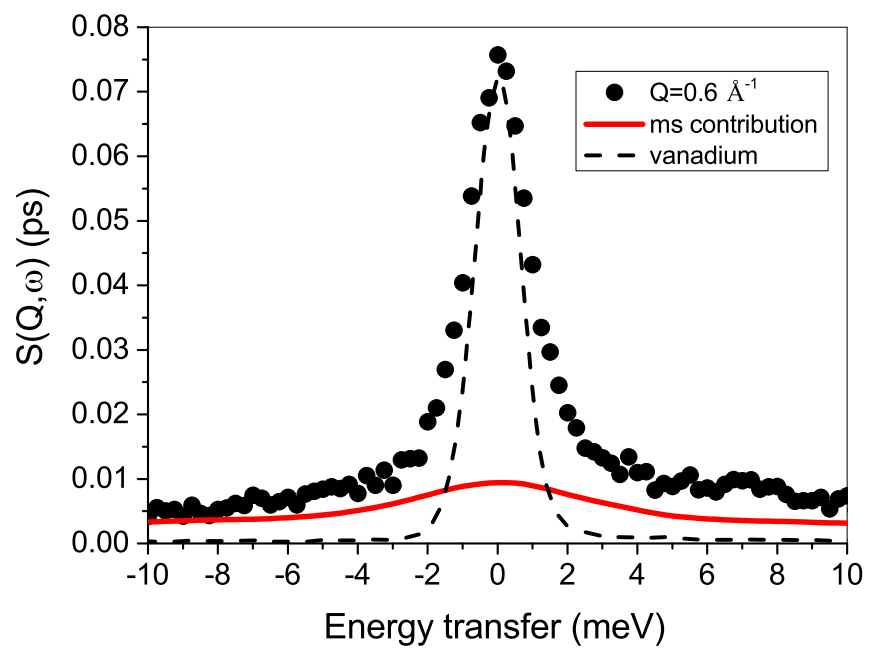

FIG. 1. (Color online) The multiple scattering noncorrected sample spectrum and a vanadium spectrum are plotted. Included is also the calculated two-times scattering neutron signal.

vectors from a slope of $1 \times 10^{-4}$ to $9 \times 10^{-4}$ at the smallest $Q$ vector $Q=0.2 \AA^{-1}$. The resulting spectra have been fitted with models as described in Sec. II. The fit model was convoluted with the resolution of the instrument represented by a Gaussian with FWHM $=1.6 \mathrm{meV}$.

Further information about the collective excitations can be obtained from the current correlation functions. These have been calculated according to Eq. (14) and are plotted in Fig. 2. Two inelastic excitation ridges are dispersing linearly and denoting the acoustic excitations. At the largest wave vector the spectrum broadens and indicates further relaxation processes.

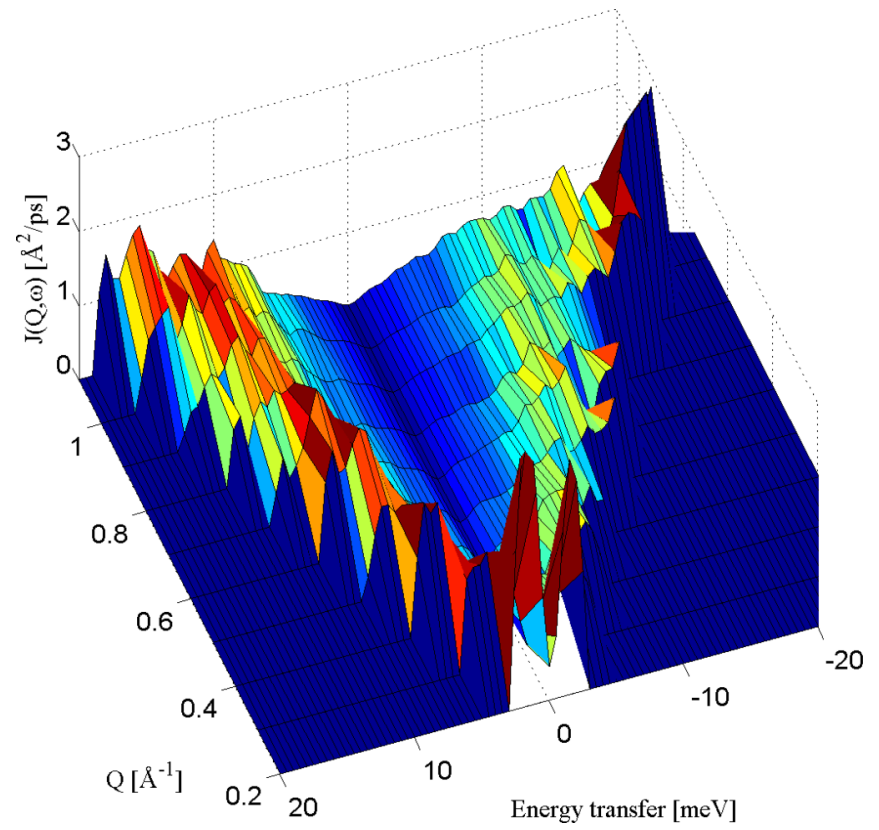

FIG. 2. (Color online) The longitudinal current spectra $J(Q, \omega)$ are plotted. Color coding defines the intensity scale: red (bright) to blue (dark) corresponds to high to low intensity.

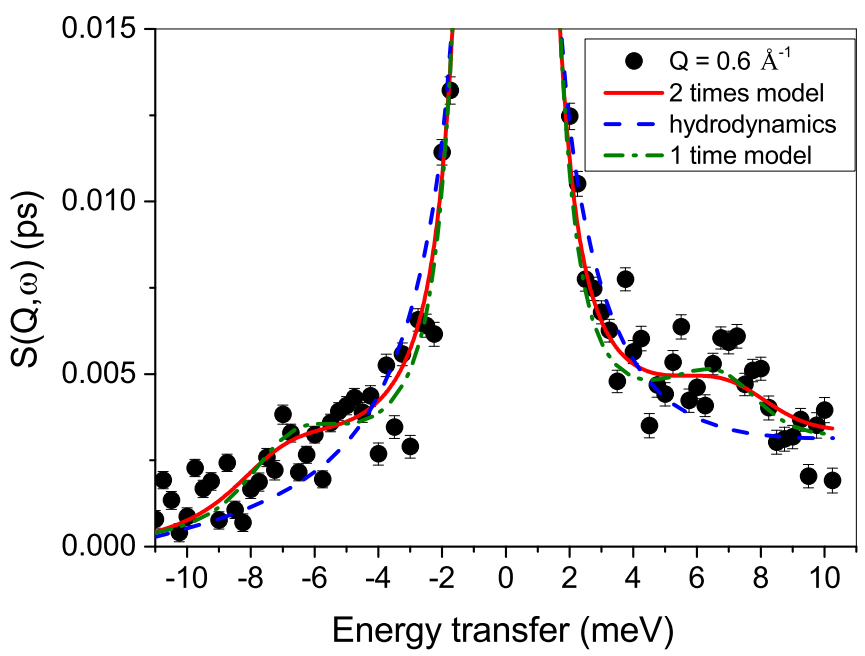

FIG. 3. (Color online) The spectrum for the wave vector $Q=$ $0.6 \AA^{-1}$ is plotted (dots). The results of three fit models are included: the hydrodynamic model (dashed), the single time viscoelastic model (dash-dot), and the two-relaxation-times model (line).

\section{RESULTS AND DISCUSSION}

In Fig. 3 we show the spectrum as measured with the spectrometer at $Q=0.6 \AA^{-1}$. Included are the results of the three fit models. Obviously, the hydrodynamic model Eq. (23) is not able to fit the data at this microscopic momentum transfer. However, both viscoelastic models deliver a good account of the data. The fit with two relaxations times for the memory functions has a smaller $\chi^{2}$ (reduced $\left.\chi^{2}=1.2 \times 10^{-6}\right)$ compared to the single relaxation time model (reduced $\chi^{2}=1.28 \times 10^{-6}$ ). However, the fitted $\omega_{l}$ value is not strongly influenced. It changes from $\omega_{l}=8 \pm$ $0.5 \mathrm{meV}$ with the two-times model to $\omega_{l}=7.3 \pm 0.4 \mathrm{meV}$. This behavior is expected because the additional relaxation time provides an additional degree of freedom for the fit of the strong Rayleigh line and hence fewer constraints for the excitation frequency fit parameter. From the fit results we conclude that the two-relaxation-times model delivers a satisfactorily good fit to the data. An inclusion of another relaxation term in the memory function is not justified due to the energy range and the statistical quality of our data.

Figure 4 shows a selection of spectra. Included is the fit result with the two-times viscoelastic model. In Fig. 4(a) we added the sloping background contribution separately. The insets emphasize the inelastic region with the respective inelastic excitations. The absolute values show a fair agreement with the corresponding intensities from Price and Copley [26]. At $Q=0.8 \AA^{-1}$ we get a peak value at zero energy transfer of $S(Q, \omega=0)=0.7 \mathrm{ps}$, which compares fairly well with the previous data which show $S\left(Q=0.8 \AA^{-1}, \omega=0\right) \approx 0.6$ ps. The inelastic contributions are small in comparison with the dominant Rayleigh line. However, the insets demonstrate quite clearly that propagating excitations exist, which are broadened due to damping.

First we will discuss the inelastic peak positions before we go into detail about the whole line shape. A fit with the two-times relaxation model Eq. (22) allowed us to determine the dispersion curve $\omega_{l}(Q)$. For the smallest $Q$ vectors 

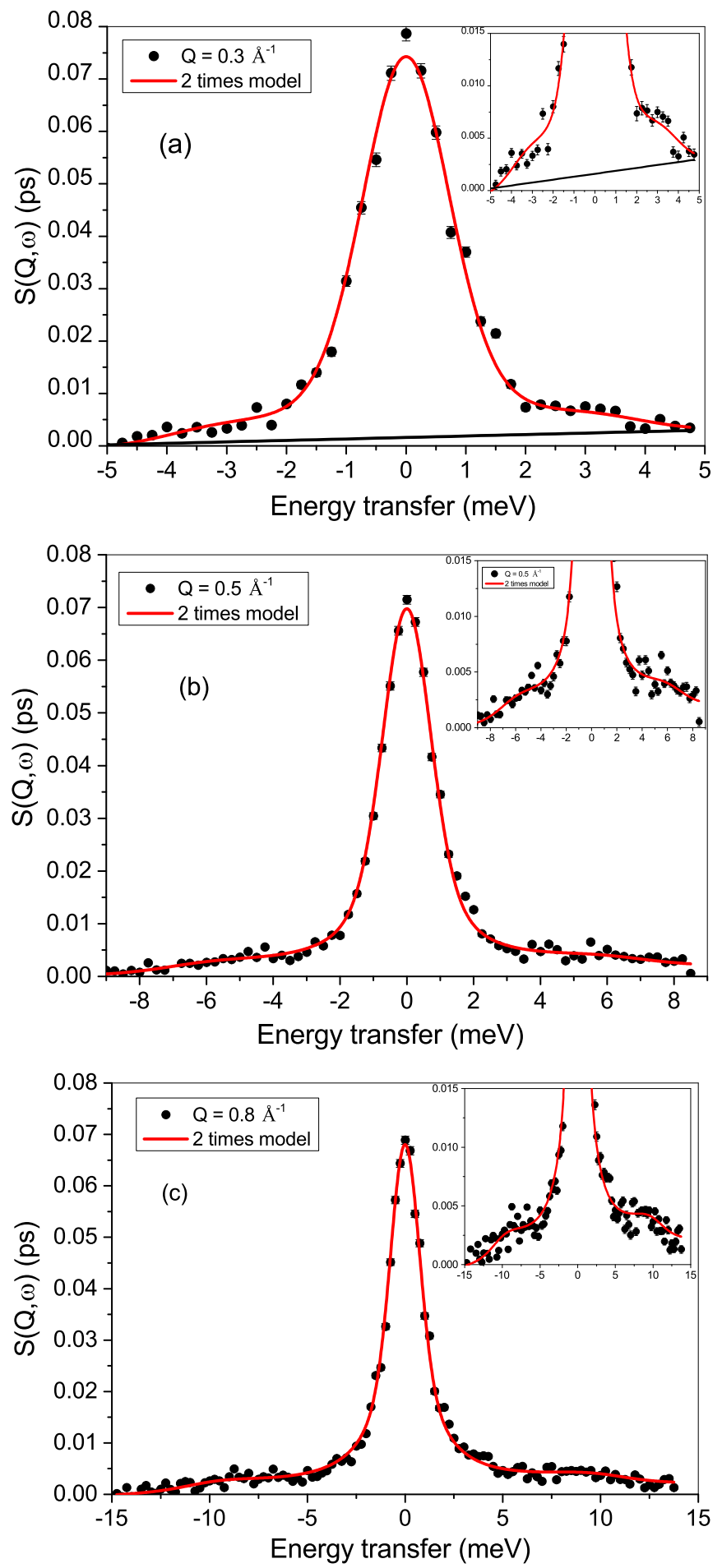

FIG. 4. (Color online) The fully corrected spectra are shown for three $Q$ vectors. Included are fits (lines) with the two-times viscoelastic model according to Eq. (22). The insets focus on the inelastic range of energies. Note the different abscissae. In plot (a) the sloping background contribution is shown.

$Q=0.2 \AA^{-1}$ and $Q=0.3 \AA^{-1}$, it was necessary to fix the thermal relaxation rate $\Gamma_{2}=0 \mathrm{meV}$ for a converging fit. Otherwise, all five fit parameters of that model were varied. The fit results for $\omega_{l}$ are plotted in Fig. 5. We observe the typical dispersive behavior of acoustic-type excitations. The

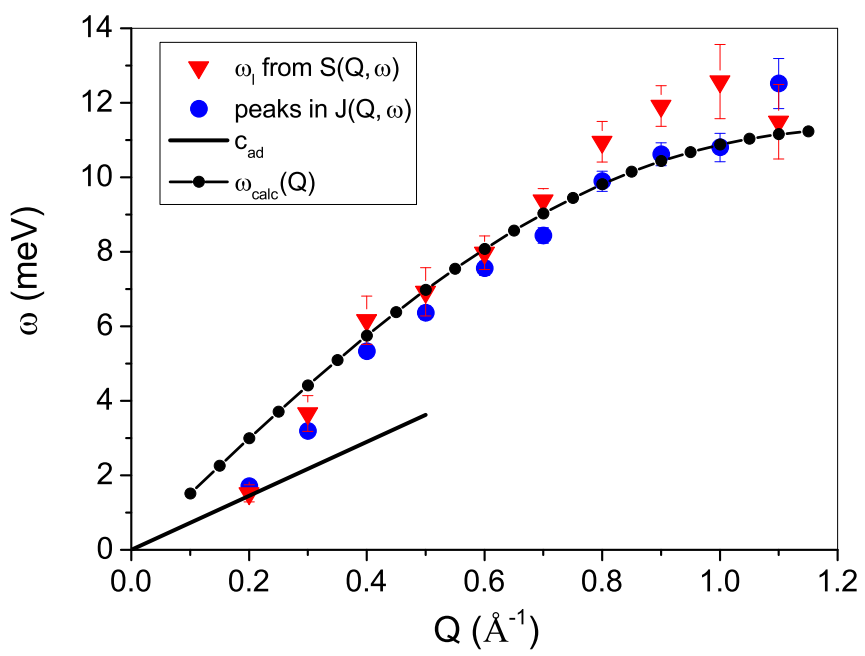

FIG. 5. (Color online) Dispersion relations for molten $\mathrm{RbBr}$. Triangles are the $\omega_{l}$ values from the two-times fit, and circles are the peak values from the current spectra $J(Q, \omega)$. The line with dots is the result for the $\omega_{\text {calc }}(Q)$ calculation according to Eq. (18) and the full line indicates the adiabatic sound velocity.

peak positions of the longitudinal current spectra $J(Q, \omega)$, obtained through a simple fit to the inelastic peak positions, are included into the figure. They agree quite well with the fit results from the two-times viscoelastic model fit. The line with dots is the calculated result for $\omega_{l}(Q)$ using Eq. (18). An Einstein frequency $\omega_{E}=12 \times 10^{12} \mathrm{ps}^{-1}$ was used and for the relaxation time $\tau$ the Lovesey recipe Eq. (24) was applied. The overall good agreement between calculated and experimental data shows that the single-relaxation-time model is a good description for the dispersion of the inelastic excitations. At $Q<0.4 \AA^{-1}$, the excitation values obtained from both $S(Q, \omega)$ and $J(Q, \omega)$ deviate from the viscoelastic behavior and the transition to a hydrodynamics (adiabatic) region is fully completed at $Q=0.2 \AA^{-1}$. In previous experiments on molten $\mathrm{NaCl}$ and $\mathrm{CsCl}$ using inelastic x-ray scattering the derived excitations approached the hydrodynamic values at the smallest resolvable wave vector around $Q \approx 0.2 \AA^{-1}$ [29]. At that time the excitation frequencies were determined with a damped harmonic oscillator model and we refer the reader to Ref. [49] for a formal comparison of fit parameters from different model functions. Our results on $\mathrm{RbBr}$ confirm these previous results for a transition to hydrodynamics.

In Fig. 6 the phase velocities $c=\frac{\omega}{Q}$ of the excitations are plotted. The phase velocity exceeds the adiabatic value $\left(c_{\mathrm{ad}}=\right.$ $1100 \mathrm{~m} / \mathrm{s}$ [56]) by nearly a factor 2 at $Q \geqslant 0.4 \AA^{-1}$. The phase velocity c approaches the hydrodynamic value at $Q=$ $0.2 \AA^{-1}$. The calculated values $c_{\text {calc }}=\omega_{\text {calc }} / Q$ describe quite well the fitted phase velocities, except at wave vectors $Q<$ $0.4 \AA^{-1}$.

An increase of the sound velocity above the adiabatic value has been observed in nearly all liquids and is called positive dispersion, even if an increase of up to $90 \%$, as found for $\mathrm{RbBr}$, is not common. For example, in liquid alkali metals a positive dispersion of about $15-20 \%$ has been observed [2,45,57], whereas for more complex liquid metals like aluminum and mercury enhancements of up to $50 \%$ have been reported [58,59], although the method to 


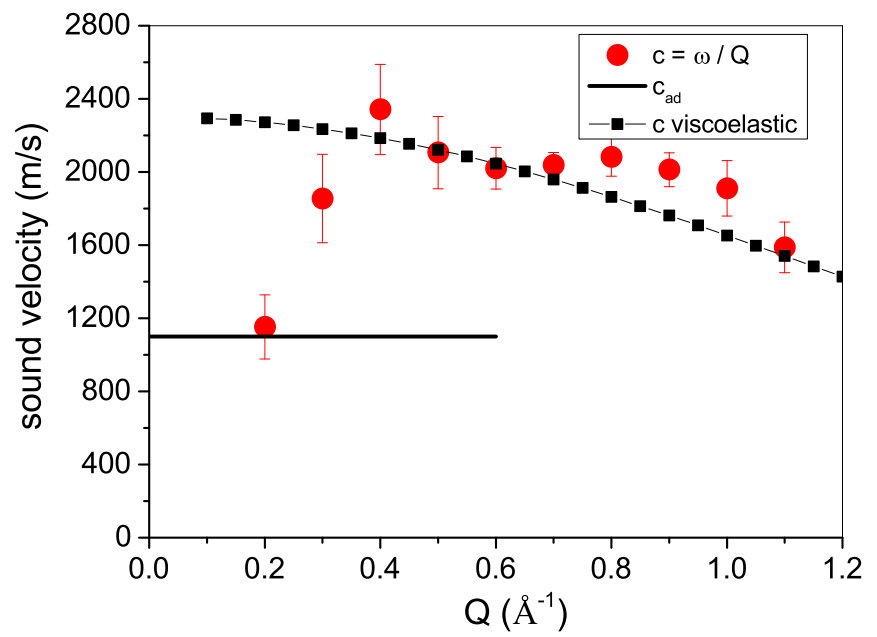

FIG. 6. (Color online) Phase velocity of molten $\mathrm{RbBr}$. The line depicts the adiabatic sound velocity. Included are calculated $c_{\text {calc }}=$ $\omega_{\text {calc }} / Q$ values using Eq. (18) as an approximation for the mode frequency.

determine the excitation frequency influences the outcome to some extent. This enhancement in frequency has been related to a viscoelastic reaction of the liquid when at high frequencies $(\omega \tau>>1)$ the liquid reacts more solidlike than at low frequencies [42]. In molten salts a much larger enhancement of the phase velocity above the adiabatic sound velocity has been observed. For example, in molten $\mathrm{NaCl}, \mathrm{KCl}, \mathrm{CsCl}$, and $\mathrm{NaI}$ the observed sound velocities exceeded the adiabatic ones by about 50-70\% [29-32]. Early evidence from MD simulation for this frequency enhancement was obtained for molten $\mathrm{RbBr}$, where a sound velocity $c=2000 \mathrm{~m} / \mathrm{s}$ from calculated current spectra was reported [34]. An enhancement of the sound velocity for the longitudinal acoustic-type excitations of $60 \%$ was reported by Ciccotti et al. in a simulation on molten $\mathrm{NaCl}$ [21]. Later simulations confirmed the large enhancements for the velocity of the propagation of sound with $c=3294 \mathrm{~m} / \mathrm{s}$ for molten $\mathrm{NaCl}$ and $c=1930 \mathrm{~m} / \mathrm{s}$ for $\mathrm{NaI}$ compared to the adiabatic sound velocities of $c_{\mathrm{ad}}=1750 \mathrm{~m} / \mathrm{s}$ and $c_{\text {ad }}=1150 \mathrm{~m} / \mathrm{s}$ for $\mathrm{NaCl}$ and $\mathrm{NaI}$, respectively [60]. To summarize, simulation results are consistent with the large positive dispersion in molten salts observed in scattering experiments. On a theoretical ground, the importance of obtaining an experimental determination of the dispersion curves relies on the need to measure the enhancement of the sound velocity and its relation with the value of the Einstein frequency [61], which itself is determined by the curvature of the potential at next-neighbor distance. In a Coulomb fluid that curvature is much stronger than in alkali metals with their quite harmonic potentials.

However, there are not many MD simulations covering the wave-number range towards hydrodynamics, because of the large box size necessary for these simulations. Interestingly, a simulation on molten NaI by Alcaraz and Trullas reports for one of the tested potentials a sound velocity at $Q=0.175 \AA^{-1}$, only $10 \%$ larger than the adiabatic sound velocity [24] and hence an indication for a transition to hydrodynamics at the same wave-number range.

From the two-relaxation-times fit we obtain the structural relaxation time $\tau_{L}(Q)=1 / \Gamma_{1}(Q)$ and the thermal relaxation

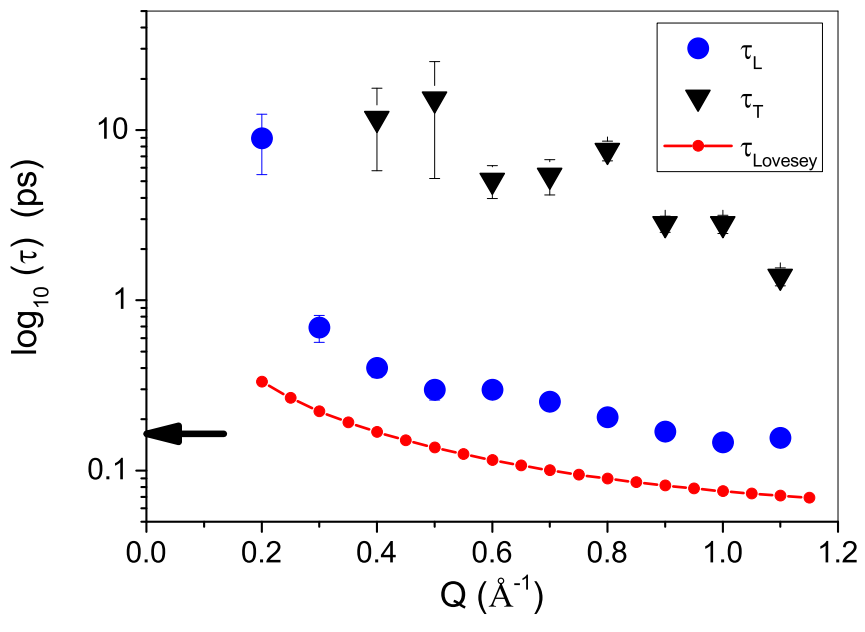

FIG. 7. (Color online) Relaxation times $\tau_{L}$ and $\tau_{T}$ of the fit with the two-times viscoelastic model is presented on a logarithmic scale. The arrow indicates the hydrodynamic limit. Included is also the calculated relaxation time $\tau_{\text {Lovesey }}$ for the viscoelastic model according to Eq. (24).

time $\tau_{T}(Q)=1 / \Gamma_{2}(Q)$. These values are plotted in Fig. 7 on a logarithmic scale. The relaxation time for the thermal process is about an order of magnitude slower than the viscoelastic relaxation time $\tau_{L}(Q)$. Note that the thermal relaxation rate $\Gamma_{2}(Q)$ is, for all $Q$ vectors, much smaller than the energy resolution. Such a slow process will improve the fit of the quasielastic line shape as it already became obvious in Fig. 3. A similar large separation in the relaxation times was observed with a two-relaxation-times fit to the data of liquid lithium [62]. Towards small $Q$ vectors the relaxation time $\tau_{L}(Q)$ increases strongly. Included in the figure is the prediction from Lovesey's recipe for the relaxation time [43]. The arrow in the figure indicates the $Q \rightarrow 0$ limit of the viscoelastic prediction [42]:

$$
\tau_{Q \rightarrow 0}=\frac{\eta_{L} / n m}{c_{L}^{2}-c_{\mathrm{ad}}^{2}} .
$$

With $\eta_{L}=1.2610^{-3}$ Pa s and a mass density $\rho=2.68 \mathrm{~g} / \mathrm{cm}^{3}$ [28] we obtain $\tau_{Q \rightarrow 0}=0.17 \mathrm{ps}$.

At the smallest wave vector $Q=0.2 \AA^{-1}$ the fitted $\tau_{L}$ increases dramatically and differs from the $\tau_{\text {Lovesey }}$ calculated value and from the $Q \rightarrow 0$ prediction. This deviation in combination with a nonconverging fit is evidence that the spectral line shape of the viscoelastic model is no more suitable.

To support our supposition that hydrodynamics is still valid at the smallest $Q$ vector, we observe (see Fig. 8) that the fit with a hydrodynamics description according to Eq. (23) is indistinguishable from a viscoelastic two-times representation at $Q=0.2 \AA^{-1}$. For the latter, $\Gamma_{2}=0 \mathrm{meV}$ was fixed for a stable converging fit. This contrasts to what was found at $Q=0.6 \AA^{-1}$ (see Fig. 3). The fit delivers a $\omega_{0}^{2}=$ $1.6 \pm 0.3 \mathrm{meV}^{2}, \Gamma_{L}=0$ and a thermal diffusion constant $D_{T}=2.5 \pm 0.37 \mathrm{meV} / \AA^{-2}=3.8 \pm 0.6 \times 10^{-4} \mathrm{~cm}^{2} / \mathrm{s}$. The Brillouin frequency $\omega_{0}$ corresponds to a sound velocity of $c=960 \pm 80 \mathrm{~m} / \mathrm{s}$, in good agreement with the adiabatic sound velocity of $c_{\mathrm{ad}}=1100 \mathrm{~m} / \mathrm{s}$. The relaxation rate $\Gamma_{L}=$ 0 indicates that the damping of the excitation cannot be 


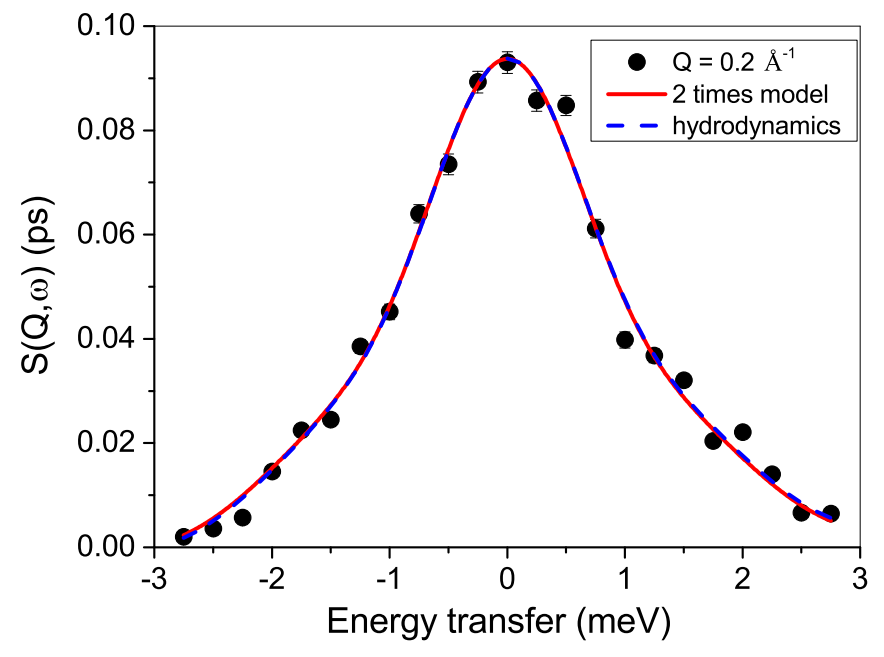

FIG. 8. (Color online) The spectrum at $Q=0.2 \AA^{-1}$ is plotted (dots). Included are fits with the hydrodynamics model and with the two-relaxation-times model, which are practically indistinguishable.

resolved by our spectral resolution. With forced Rayleigh scattering Nakazawa et al. determined experimentally thermal diffusivities of molten alkali halides [63]. For molten $\mathrm{RbBr}$ at $1031 \mathrm{~K}$ they report that $D_{T}=1.76 \times 10^{-3} \mathrm{~cm}^{2} / \mathrm{s}$. That value is about a factor 4 larger than our fit value. At larger $Q$ values the damping coefficient $\Gamma_{L}$ increases substantially to deliver an overdamped excitation (see Fig. 3).

The collective dynamics of molten alumina was studied by use of inelastic x-ray scattering [64]. For molten alumina the data analysis, based on a generalized hydrodynamics model of a sum of three Lorentzians, revealed for the small-wave-vector range a thermal diffusivity $D_{T}$ which was about 2 to 3 orders of magnitude smaller than the macroscopic expected value $D_{T}$, derived from the thermal conductivity $\lambda$. It seems that in the case of liquid alumina, the transition from a hydrodynamic to viscoelastic response occurs at much smaller wave vectors and that the oxide melts show herein a difference to molten alkali halides [65]. For molten $\mathrm{RbBr}$ the hydrodynamic $\Gamma=D_{T} Q^{2}$ law deviates by only a factor 4 at the smallest $Q$ vector. A perfect agreement is not expected since $Q=0.2 \AA^{-1}$ is not yet the hydrodynamic limit and another uncertain parameter is the $Q$ dependence of the specific heat ratio $\gamma=1.5$, which was kept constant during our analysis.

The transition from hydrodynamics to microscopic dynamics was studied previously on argon gas [35]. That study concluded that the collective dynamics of the gas at room temperature can be described by a hydrodynamics approach based on kinetic theory up to wave vectors of $Q \approx 0.1 \AA^{-1}$. The wave-vector dependence of the fit parameters follow the hydrodynamics predictions in this $Q$ range. At higher densities deviations from the linearized hydrodynamics prediction have been observed. Simulations of a rare gas mixture concluded that the collective dynamics followed hydrodynamics up to a wave vector of $Q=0.2 \AA^{-1}$ [37], which agrees very well with our observations in molten RbBr.

Within the viscoelastic model the transition to highfrequency sound propagation occurs when $\omega \tau \approx 1$ is valid. Deviations from the viscoelastic description appear at a wave vector $Q \approx 0.3 \AA^{-1}$ in our data. At that $Q$ vector we obtain an excitation frequency of $\omega \approx 4.5 \mathrm{ps}^{-1}$, which delivers a corresponding relaxation time $\tau=0.22 \mathrm{ps}$, in good agreement with $\tau_{Q \rightarrow 0}=0.17 \mathrm{ps}$, the calculated limit value for the hydrodynamic regime. That estimate supports our supposition that at the smallest observed excitation frequency at $Q=0.2 \AA^{-1}$ the transition to hydrodynamics has occurred.

\section{CONCLUSIONS}

An inelastic neutron-scattering experiment was performed on molten $\mathrm{RbBr}$ using the dedicated Brillouin spectrometer BRISP. The choice of a binary liquid composed of components having similar scattering lengths allowed us to focus on the collective acoustic-like dynamics at small wave vectors in order to study the departure from the hydrodynamics regime. Raw data were carefully corrected and a refined subtraction of the multiple-scattering contribution was performed. The spectra then reveal the presence of dispersing acoustic modes around a dominating quasielastic line. A two-times viscoelastic model fits the spectra sufficiently well and delivers an acoustic dispersion with a large positive dispersion of about $100 \%$ above the values expected from the adiabatic sound velocity. This frequency analysis was supported by the inspection of the longitudinal current spectra. However, at the smallest wave vector the dispersion approaches the hydrodynamic values. Furthermore, the derived structural relaxation time increases strongly beyond the limit value for long wavelengths in this wave-vector range. A fit with a purely hydrodynamic model works well at $Q \approx 0.2 \AA^{-1}$ and underlines the conclusion that at that wave vector the transition to hydrodynamics occurs in molten $\mathrm{RbBr}$. Within the viscoelastic picture the transition from viscoelastic propagation of acoustic modes to hydrodynamic adiabatic velocity of sound will happen when the excitation frequency is similar to the inverse time of structural relaxations. We derive a relaxation time at the transition in good agreement with the hydrodynamic limit of the viscoelastic model supporting the conclusion that we are observing the transition to ordinary hydrodynamics in molten $\mathrm{RbBr}$. Revealing more details of the transition would need a larger kinematic range in combination with better energy resolution, demands which are mutual contradictory. It might be interesting to study the transition region from $\mathrm{GHz}$ to $\mathrm{THz}$ dynamics in the more complex counterparts of the molten salts, the ionic liquids. These organic Coulomb liquids have internal degrees of freedom and it has been suggested by MD simulations that stiff and soft domains exist [66], which may provide a spatial origin for a viscoelastic response.

\section{ACKNOWLEDGMENTS}

We are grateful to Chr. Morkel for a critical reading of the manuscript and encouraging discussions. A. Laloni is acknowledged for his valuable assistance to set up the instrument and to run the experiment. We thank the Institut Laue-Langevin for the provision of beam time. This work was partly supported by the Science and Technology Facilities Council, STFC. 


\section{APPENDIX}

In this Appendix we will provide more details in the derivation of Eq. (22). We start with the continued fraction representation:

$$
\frac{F(Q, z)}{S(Q)}=\frac{1}{z+\frac{\omega_{0}^{2}}{z+K(Q, z)}}
$$

where $\omega_{0}^{2}$ is the normalized second frequency moment of $S(Q, \omega)$ and $K(Q, z)$ is the Laplace transform of the memory function $K(Q, t)$. With two relaxation channels to the memory function:

$$
K(Q, t)=\left(\omega_{l}^{2}-\gamma \omega_{0}^{2}\right) \exp [-t / \tau(Q)]+(\gamma-1) \omega_{0}^{2} \exp \left(-D_{T} Q^{2} t\right)
$$

and after a Laplace transform we obtain:

$$
\frac{F(Q, z)}{S(Q)}=\frac{1}{z+\frac{\omega_{0}^{2}}{z+\frac{\Delta_{1}}{z+\Gamma_{1}}+\frac{\Delta_{2}}{z+\Gamma_{2}}}}
$$

with $\Delta_{1}=\omega_{l}^{2}-\gamma \omega_{0}^{2}, \Delta_{2}=(\gamma-1) \omega_{0}^{2}, \Gamma_{1}=1 / \tau(Q)$, and $\Gamma_{2}=D_{T} Q^{2}$. Here $\tau(Q)$ is the structural, viscoelastic relaxation time and $\tau_{T}=1 / \Gamma_{2}$ is the thermal relaxation time. This fraction can be simplified:

$$
\frac{F(Q, z)}{S(Q)}=\frac{1}{z+\frac{\omega_{0}^{2}\left(z+\Gamma_{1}\right)\left(z+\Gamma_{2}\right)}{z\left(z+\Gamma_{1}\right)\left(z+\Gamma_{2}\right)+\Delta_{1}\left(z+\Gamma_{2}\right)+\Delta_{2}\left(z+\Gamma_{1}\right)}}=\frac{z\left(z+\Gamma_{1}\right)\left(z+\Gamma_{2}\right)+\Delta_{1}\left(z+\Gamma_{2}\right)+\Delta_{2}\left(z+\Gamma_{1}\right)}{z^{2}\left(z+\Gamma_{1}\right)\left(z+\Gamma_{2}\right)+z \Delta_{1}\left(z+\Gamma_{2}\right)+z \Delta_{2}\left(z+\Gamma_{1}\right)+\omega_{0}^{2}\left(z+\Gamma_{1}\right)\left(z+\Gamma_{2}\right)} .
$$

The numerator can now be written as

$$
z^{3}+z^{2}\left(\Gamma_{1}+\Gamma_{2}\right)+z\left(\Gamma_{1} \Gamma_{2}+\Delta_{1}+\Delta_{2}\right)+\left(\Delta_{1} \Gamma_{2}+\Delta_{2} \Gamma_{1}\right)=z^{3}+z^{2} f_{1}+z f_{2}+f_{3}
$$

and the denominator as

$$
\begin{aligned}
z^{4} & +z^{3}\left(\Gamma_{1}+\Gamma_{2}\right)+z^{2}\left(\Gamma_{1} \Gamma_{2}+\Delta_{1}+\Delta_{1}+\omega_{0}^{2}\right)+z\left(\Delta_{1} \Gamma_{2}+\Delta_{2} \Gamma_{1}+\omega_{0}^{2} \Gamma_{1}+\omega_{0}^{2} \Gamma_{2}\right)+\left(\omega_{0}^{2} \Gamma_{2} \Gamma_{1}\right) \\
& =z^{4}+z^{3} g_{1}+z^{2} g_{2}+z g_{3}+g_{4} .
\end{aligned}
$$

Note that $f_{1}=g_{1}$. Then we arrive at the following expression for the two-times viscoelastic model:

$$
\frac{F(Q, z)}{S(Q)}=\frac{z^{3}+z^{2} f_{1}+z f_{2}+f_{3}}{z^{4}+z^{3} f_{1}+z^{2} g_{2}+z g_{3}+g_{4}} .
$$

To get $S(Q, \omega)$ we need to obtain the real part of $F(Q, z)$ :

$$
\frac{S(Q, \omega)}{S(Q)}=\frac{1}{\pi} \operatorname{Re}\left[\frac{F(Q, z=i \omega)}{S(Q)}\right]=\frac{1}{\pi} \frac{-\omega^{2} f_{1}+f_{3}-i\left(\omega^{3}-\omega f_{2}\right)}{\omega^{4}-\omega^{2} g_{2}+g_{4}-i\left(\omega^{3} f_{1}-\omega g_{3}\right)} .
$$

Multiplying with the complex conjugate of the denominator we get for the denominator:

$$
\left(\omega^{4}-\omega^{2} g_{2}+g_{4}\right)^{2}+\left(\omega^{3} f_{1}-\omega g_{3}\right)^{2}=\omega^{8}+\omega^{6}\left(f_{1}^{2}-2 g_{2}\right)+\omega^{4}\left(g_{2}^{2}+2 g_{4}-2 f_{1} g_{3}\right)+\omega^{2}\left(g_{3}^{2}-2 g_{2} g_{4}\right)+g_{4}^{2}
$$

and for the real part of the numerator:

$\left(-\omega^{2} f_{1}+f_{3}\right)\left(\omega^{4}-\omega^{2} g_{2}+g_{4}\right)+\left(\omega^{3}-\omega f_{2}\right)\left(\omega^{3} f_{1}-\omega g_{3}\right)=\omega^{4}\left(f_{1} g_{2}+f_{3}-g_{3}-f_{1} f_{2}\right)+\omega^{2}\left(f_{2} g_{3}-f_{1} g_{4}-f_{3} g_{2}\right)+f_{3} g_{4}$.

The fraction of both terms is the end result given in Eq. (22) and a similar derivation leads us to Eq. (23).

[1] Chr. Morkel, Chr. Gronemeyer, W. Gläser, and J. Bosse, Phys. Rev. Lett. 58, 1873 (1987).

[2] T. Bodensteiner, Chr. Morkel, W. Gläser, and B. Dorner, Phys. Rev. A 45, 5709 (1992).

[3] T. Scopigno, G. Ruocco, and F. Sette, Rev. Mod. Phys. 77, 881 (2005).

[4] F. Demmel, G. Heusel, I. Waldner, J. Stride, and H. Bertagnolli, Z. Phys. Chem. 222, 1551 (2008); F. J. Bermejo, J. W. Taylor, S. E. McLain, I. Bustinduy, J. F. C. Turner, M. D. Ruiz-Martin, C. Cabrillo, and R. Fernandez-Perea, Phys. Rev. Lett. 96, 235501 (2006).
[5] F. Demmel, S. Hosokawa, W.-C. Pilgrim, and S. Tsutsui, Nucl. Instrum. Methods B 238, 98 (2005).

[6] L. E. Bove, F. Formisano, E. Guarini, A. Ivanov, C. Petrillo, and F. Sacchetti, Europhys. Lett. 79, 16002 (2007).

[7] S. Jahn and J. B. Suck, Phys. Rev. Lett. 92, 185507 (2004).

[8] J. Bosse, G. Jacucci, M. Ronchetti, and W. Schirmacher, Phys. Rev. Lett. 57, 3277 (1986).

[9] J. Teixeira, M. C. Bellissent-Funel, S. H. Chen, and B. Dorner, Phys. Rev. Lett. 54, 2681 (1985); F. Sette, G. Ruocco, M. Krisch, U. Bergmann, C. Masciovecchio, V. Mazzacurati, G. Signorelli, and R. Verbeni, ibid. 75, 850 (1995); F. Sette, 
G. Ruocco, M. Krisch, C. Masciovecchio, R. Verbeni, and U. Bergmann, ibid. 77, 83 (1996).

[10] F. Sacchetti, J. B. Suck, C. Petrillo, and B. Dorner, Phys. Rev. E 69, 061203 (2004).

[11] A. Cunsolo, C. N. Kodituwakku, F. Bencivenga, M. Frontzek, B. M. Leu, and A. H. Said, Phys. Rev. B 85, 174305 (2012).

[12] U. Balucani, G. Ruocco, A. Torcini, and R. Vallauri, Phys. Rev. E 47, 1677 (1993).

[13] A. Campa and E. G. D. Cohen, Phys. Rev. Lett. 61, 853 (1988); W. Montfrooij, P. Westerhuijs, V. O. deHaan, and I. M. deSchepper, ibid. 63, 544 (1989); P. Westerhuijs, W. Montfrooij, L. A. deGraaf, and I. M. deSchepper, Phys. Rev. A 45, 3749 (1992).

[14] N. H. March and M. P. Tosi, Coulomb Liquids (Academic Press, San Diego, CA, 1984).

[15] M. Rovere and M. P. Tosi, Rep. Prog. Phys. 49, 1001 (1986).

[16] J. P. Hansen and I. McDonald, Theory of Simple Liquids (Academic Press, London, 2006).

[17] F. G. Edwards, J. E. Enderby, R. A. Howe, and D. I. Page, J. Phys. C 8, 3483 (1975); J. Y. Derrien and J. Dupuy, J. de Phys. 36, 191 (1975); J. Locke, S. Messoloras, R. J. Stewart, R. L. McGreevy, and E. W. J. Mitchell, Phil. Mag. B 51, 301 (1985); M. A. Howe, R. L. McGreevy, and W. S. Howells, J. Phys.: Condens. Matter 1, 3433 (1989).

[18] R. L. McGreevy, in Solid State Physics, edited by H. Ehrenreich and D. Turnbull (Academic Press, Orlando, 1987), Vol. 40, p. 247.

[19] J. P. Hansen and I. R. McDonald, Phys. Rev. A 11, 2111 (1975).

[20] E. M. Adams, I. R. McDonald, and K. Singer, Proc. R. Soc. London A 357, 37 (1977).

[21] G. Ciccotti, G. Jacucci, and I. R. McDonald, Phys. Rev. A 13, 426 (1976).

[22] M. Dixon, Philos. Mag. B 47, 531 (1983); 48, 13 (1983).

[23] R. A. Cowley, W. Cochran, B. N. Brockhouse, and A. D. B. Woods, Phys. Rev. 131, 1030 (1963).

[24] O. Alcaraz and J. Trullàs, J. Mol. Liquids 136, 227 (2007).

[25] R. L. McGreevy, E. W. J. Mitchell, and F. M. A. Margaca, J. Phys. C 17, 775 (1984).

[26] D. L. Price and J. R. D. Copley, Phys. Rev. A 11, 2124 (1975).

[27] S. L. Qiu, R. A. J. Bunten, M. Dutta, E. W. J. Mitchell, and H. Z. Cummins, Phys. Rev. B 31, 2456 (1985).

[28] G. J. Janz, Molten Salts Handbook (Academic Press, New York, 1967).

[29] F. Demmel, S. Hosokawa, M. Lorenzen, and W. C. Pilgrim, Phys. Rev. B 69, 012203 (2004).

[30] M. Inui, S. Hosokawa, Y. Kajihara, K. Matsuda, S. Tsutsui, and A. Q. R. Baron, J. Phys.: Condens. Matter 19, 466110 (2007).

[31] F. Demmel, S. Hosokawa, and W. C. Pilgrim, J. Alloys Comp. 452, 143 (2008).

[32] S. Hosokawa, F. Demmel, W. C. Pilgrim, M. Inui, S. Tsutsui, and A. Baron, Electrochemistry 77, 608 (2009).

[33] P. Chieux, J. Dupuy-Philon, J. F. Jal, C. Morkel, and J. B. Suck, J. Phys.: Condens. Matter 6, A235 (1994).

[34] J. R. D. Copley and A. Rahman, Phys. Rev. A 13, 2276 (1976).

[35] U. Bafile, P. Verkerk, F. Barocchi, L. A. de Graaf, J.-B. Suck, and H. Mutka, Phys. Rev. Lett. 65, 2394 (1990).
[36] U. Bafile, P. Verkerk, E. Guarini, and F. Barocchi, Phys. Rev. Lett. 86, 1019 (2001).

[37] M. Sampoli, U. Bafile, E. Guarini, and F. Barocchi, Phys. Rev. Lett. 88, 085502 (2002).

[38] N. W. Ashcroft and D. C. Langreth, Phys. Rev. 156, 685 (1967).

[39] V. F. Sears, Neutron News 3(3), 26 (1992).

[40] L. Köster, K. Knopf, and W. Waschkowski, Z. Phys. A 301, 215 (1981).

[41] J. P. Boon and S. Yip, Molecular Hydrodynamics (McGraw-Hill, New York, 1980).

[42] U. Balucani and M. Zoppi, Dynamics of the Liquid State (Clarendon Press, Oxford, 1994).

[43] S. W. Lovesey, J. Phys. C 4, 3057 (1971).

[44] J. Hubbard and J. L. Beeby, J. Phys. C 2, 556 (1969).

[45] F. Demmel, D. Pasqualini, and C. Morkel, Phys. Rev. B 74, 184207 (2006).

[46] D. Pasqualini, R. Vallauri, F. Demmel, Chr. Morkel, and U. Balucani, J. Non-Cryst. Solids 250-252, 76 (1999).

[47] D. Levesque, L. Verlet, and J. Kurkijarvi, Phys. Rev. A 7, 1690 (1973).

[48] J. M. Bockris and N. E. Richards, Proc. Roy. Soc. A 241, 44 (1957).

[49] U. Bafile, E. Guarini, and F. Barocchi, Phys. Rev. E 73, 061203 (2006).

[50] L. D. Landau and E. M. Lifshitz, Fluid Mechanics (Pergamon Press, London, 1959).

[51] F. Formisano, A. De Francesco, E. Guarini, A. Laloni, A. Orecchini, C. Petrillo, W. C. Pilgrim, D. Russo, and F. Sacchetti, J. Phys. Soc. Jpn. 82, SA028 (2013); D. Aisa et al., Nucl. Instrum. Methods A 544, 620 (2005); http://www.ill.eu/brisp.

[52] E. Guarini et al., Phys. Rev. B 88, 104201 (2013).

[53] C. Petrillo, E. Guarini, F. Formisano, F. Sacchetti, E. Babucci, and C. Campeggi, Nucl. Instrum. Methods A 489, 304 (2002).

[54] P. D. Mitev, M. Saito, and Y. Waseda, J. Non-Cryst. Solids 312-314, 443 (2002).

[55] http://www.ill.eu/instruments-support/computing-for-science/ cs-software/all-software/lamp/.

[56] S. Sternberg and V. Vasilescu, J. Chem. Thermodynam. 3, 877 (1971).

[57] W. C. Pilgrim, S. Hosokawa, H. Saggau, H. Sinn, and E. Burkel, J. Non-Cryst. Solids 250-252, 96 (1999).

[58] T. Scopigno, U. Balucani, G. Ruocco, and F. Sette, Phys. Rev. E 63, 011210 (2000).

[59] L. E. Bove, F. Sacchetti, C. Petrillo, B. Dorner, F. Formisano, and F. Barocchi, Phys. Rev. Lett. 87, 215504 (2001).

[60] T. Bryk and I. Mryglod, Phys. Rev. B 71, 132202 (2005).

[61] U. Balucani and M. Zoppi, Dynamics of the Liquid State (Clarendon Press, Oxford, 1994), p. 241.

[62] T. Scopigno, U. Balucani, G. Ruocco, and F. Sette, Phys. Rev. Lett. 85, 4076 (2000).

[63] N. Nakazawa, Y. Nagasaka, and A. Nagashima, Int. J. Thermodynam. 13, 753 (1992).

[64] H. Sinn, B. Glorieux, L. Hennet, A. Alatas, M. Hu, E. E. Alp, F. J. Bermejo, D. L. Price, and M. L. Saboungi, Science 299, 2047 (2003).

[65] S. Jahn and P. Madden, Condensed Matter Phys. 11, 169 (2008).

[66] M. C. Ribeiro, J. Chem. Phys. 139, 114505 (2013). 